\title{
BROWDER-LIVESAY FILTRATIONS AND THE EXAMPLE OF CAPPELL AND SHANESON
}

\author{
F. Hegenbarth, Y. V. Muranov, and D. Repovš
}

\begin{abstract}
Let $M^{3}$ be a 3-dimensional manifold with fundamental group $\pi_{1}(M)$ which contains a quaternion subgroup $Q$ of order 8. In 1979 Cappell and Shaneson constructed a nontrivial normal map $f: M^{3} \times T^{2} \rightarrow M^{3} \times S^{2}$ which cannot be detected by simply connected surgery obstructions along submanifolds of codimension 0 , 1 , or 2 , but it can be detected by the codimension 3 Kervaire-Arf invariant. The proof of non-triviality of $\sigma(f) \in L_{5}\left(\pi_{1}(M)\right)$ is based on consideration of a BrowderLivesay filtration of a manifold $X$ with $\pi_{1}(X) \cong \pi_{1}(M)$. For a Browder-Livesay pair $Y^{n-1} \subset X^{n}$, the restriction of a normal map to the submanifold $Y$ is given by a partial multivalued map $\Gamma: L_{n}\left(\pi_{1}(X)\right) \rightarrow$ $L_{n-1}\left(\pi_{1}(Y)\right)$, and the Browder-Livesay filtration provides an iteration $\Gamma^{n}$. This map is a basic step in the definition of the iterated Browder-Livesay invariants which give obstructions to realization of surgery obstructions by normal maps of closed manifolds.

In the present paper we prove that $\Gamma^{3}(\sigma(f))=0$ for any Browder-Livesay filtration of a manifold $X^{4 k+1}$ with $\pi_{1}(X) \cong Q$. We compute splitting obstruction groups for various inclusions $\rho \rightarrow Q$ of index 2, describe natural maps in the braids of exact sequences, and make more precise several results about surgery obstruction groups of the group $Q$.
\end{abstract}

1. Introduction. Let $M^{3}$ be a 3-dimensional manifold with fundamental group $\pi_{1}(M)$ which contains the quaternion subgroup

$$
Q=\left\{x, y \mid x^{4}=y^{2}, y x y^{-1}=x^{-1}\right\}
$$

of order 8. Consider a map

$$
f: M^{3} \times T^{2} \longrightarrow M^{3} \times S^{2}
$$

where $T^{2}=S^{1} \times S^{1}$ is the torus equipped with the Lie group invariant framing [1], and $T^{2} \longrightarrow S^{2}$ is a normal map with a nontrivial Kervaire-Arf invariant. In 1979 Cappell and Shaneson [1] proved that surgery obstruction $\sigma(f)$ is nontrivial. The nontriviality of $\sigma(f)$ cannot be detected by simply connected surgery obstructions along submanifolds of codimension 0,1 , and 2 , but it can be detected by the codimension 3 Arf-invariant (see [1] and [5]).

Recall that a pair of manifolds

$$
Y^{n-1} \subset X^{n}
$$

is called a Browder-Livesay pair if $Y$ is a codimension one locally-flat closed submanifold and the natural inclusion $\pi_{1}(Y) \rightarrow \pi_{1}(X)$ induces an isomorphism of fundamental groups. In what follows

2010 Mathematics Subject Classification. Primary 57R67, 19J25; Secondary 55T99, 58A35, 18 F25.

Key words and phrases. Capppell and Shaneson example, surgery obstruction groups, closed manifolds surgery problem, splitting obstruction groups, Browder-Livesay invariants, Browder-Livesay groups, manifolds with filtration.

Acknowledgements. The second author was supported by the CONACyT Grant 98697 and he thanks the Commission on Development and Exchange of the International Mathematical Union for the travel support. The third author was supported by the SRA grants P1-0292-0101, J1-2057-0101, and J1-4144-0101. 
we shall consider only the case when $\operatorname{dim} Y=n-1 \geq 5$. For a Browder-Livesy pair, we can write down a push-out square of fundamental groups

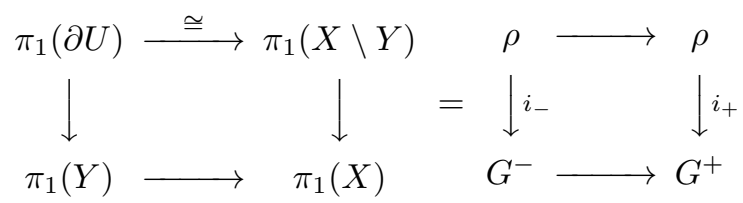

where $\partial U$ is the boundary of a tubular neighborhood of $Y$ in $X$, and $X \backslash Y$ is the closure of the complement of a tubular neighborhood. The groups in square (1.4) are equipped with orientation. The upper horizontal map and the vertical maps agree with orientations. The bottom horizontal map preserves the orientation on the images of the vertical maps which are inclusions of index 2 and reverses orientation outside of these images. We shall denote this fact by superscript " +" or "-". We shall omit this superscript if the orientation is clear from the context.

For a Browder-Livesay pair (1.3) we have a braid of exact sequences (see [3] and [8]):

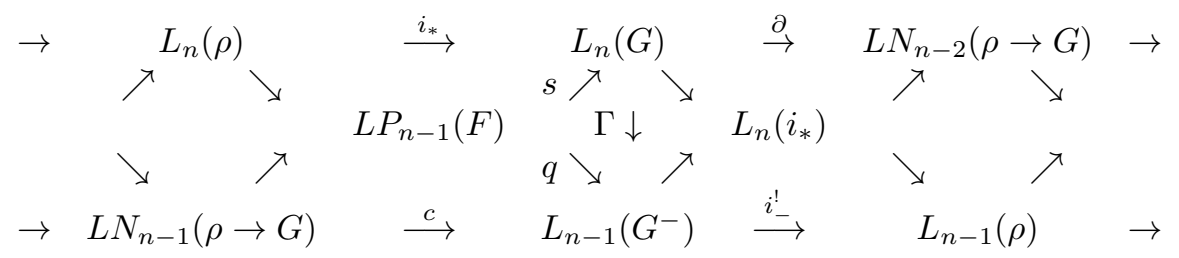

where $L P_{n-1}(F) \cong L_{n}\left(i_{-}^{!}\right)$are surgery obstruction groups for the manifold pair $(X, Y), L N_{n}(\rho \rightarrow$ $G)$ are the splitting obstruction groups, and $L_{n}\left(i_{*}\right)$ are the relative surgery obstruction groups for the inclusion map $i$ (see [3], [8] and [9]). The horizontal rows in (1.5) are chain complexes and $\Gamma$ is an isomorphism of the corresponding homology groups. The maps $s$ and $q$ are the natural forgetful maps, and the map $c$ denotes passing from surgery problem inside the manifold $X^{n}$ to an abstract surgery problem. For a normal map $f: M \rightarrow X$, the restriction to a transversal preimage

$$
\left.f\right|_{f^{-1}(Y)}: f^{-1}(Y) \rightarrow Y^{n-1}
$$

is a normal map to $Y^{n-1}$, and the corresponding map of surgery obstruction groups is given by a partial multivalued map $\Gamma: L_{n}\left(\pi_{1}(X)\right) \rightarrow L_{n-1}\left(\pi_{1}(Y)\right)$.

Let $\mathcal{X}$ be a filtration

$$
X_{k} \subset X_{k-1} \subset \cdots \subset X_{2} \subset X_{1} \subset X_{0}=X
$$

of a closed $n$-dimensional manifold $X$ by means of locally flat closed submanifolds. A filtration in (1.6) is called a Browder-Livesay filtration, if every pair of submanifolds $\left(X_{i}, X_{i+1}\right)(0 \leq i \leq k-1)$ is a Browder-Livesay pair (see [2], [5] and [7]). In what follows we shall assume that $\operatorname{dim} X_{k}=$ $n-k \geq 5$.

Let $F_{i}(0 \leq i \leq k-1)$ be a square of fundamental groups in the splitting problem for a manifold pair $\left(X_{i}, X_{i+1}\right)$ of a filtration in (1.6), $G_{i}=\pi_{1}\left(X_{i}\right)$, and $\rho_{i}=\pi_{1}\left(\partial U_{i+1}\right) \cong \pi_{1}\left(X_{i} \backslash X_{i+1}\right)$, where $\partial U_{i+1}$ is a boundary of a tubular neighborhood of $X_{i+1}$ in $X_{i}$. Then $L N_{*}\left(\rho_{i} \rightarrow G_{i}\right)$ are the splitting obstruction groups for the manifold pair $\left(X_{i}, X_{i+1}\right)$.

Every inclusion $\rho_{i} \rightarrow G_{i}$ of index 2 of oriented groups gives a commutative braid of exact sequences (1.5). Putting together central squares from these diagrams (see [4], [5] and [7] ) we obtain a commutative diagram 


\begin{tabular}{|c|c|c|c|}
\hline$\longrightarrow$ & $L_{n}\left(G_{0}\right)$ & $\stackrel{\partial_{0}}{\rightarrow}$ & $L N_{n-2}\left(\rho_{0} \rightarrow G_{0}\right)$ \\
\hline$L P_{n-1}\left(F_{0}\right)$ & $\begin{array}{l}s \nearrow{ }^{s}{ }^{\Gamma \downarrow} \\
q \searrow \nearrow r\end{array}$ & $L_{n}\left(\rho_{0} \rightarrow G_{0}\right)$ & \\
\hline$\longrightarrow$ & $\begin{array}{c}L_{n-1}\left(G_{1}\right) \\
s \nearrow \searrow p\end{array}$ & $\stackrel{\partial_{7}}{\rightarrow}$ & $L N_{n-3}\left(\rho_{1} \rightarrow G_{1}\right)$ \\
\hline$L P_{n-2}\left(F_{1}\right)$ & $q \searrow^{\Gamma \downarrow} \nearrow r$ & $L_{n-1}\left(\rho_{1} \rightarrow G_{1}\right)$ & \\
\hline$\longrightarrow$ & 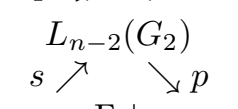 & $\stackrel{\partial_{2}}{\rightarrow}$ & $L N_{n}\left(\rho_{2} \rightarrow G_{2}\right)$ \\
\hline$L P_{n-3}\left(F_{2}\right)$ & $q \searrow_{L_{n-3}\left(G_{3}\right)}^{\Gamma \downarrow}$ & $L_{n-2}\left(\rho_{2} \rightarrow G_{2}\right)$ & \\
\hline$\longrightarrow$ & $\begin{array}{c}L_{n-k+1}\left(G_{k-1}\right) \\
s \nearrow \searrow \searrow\end{array}$ & $\stackrel{\partial_{k-1}}{\rightarrow}$ & $L N_{n-k-1}\left(\rho_{k-1} \rightarrow G_{k-1}\right)$ \\
\hline$L P_{n-k}\left(F_{k-1}\right)$ & $q \searrow^{\Gamma \downarrow}{ }_{L_{n-k}}\left(G_{k}\right)$. & $L_{n-k+1}\left(\rho_{k-1} \rightarrow G_{k-1}\right)$ & \\
\hline
\end{tabular}

In this diagram we denote by $s, q, p$, and $r$ similar maps from different diagrams. However, in what follows, it will be clear from the context which map is under consideration. Note that the groups and the maps in diagram (1.7) are defined by the subscripts taken mod 4 .

Now we recall an inductive definition of the sets

$$
\Gamma^{j}(x) \subset L_{n-j}\left(G_{j}\right) \text { for }(0 \leq j \leq k)
$$

and the iterated Browder-Livesay $j$-invariants $(1 \leq j \leq k)$ with respect to filtration (1.6) (see [5] and $[7])$.

Definition 1. Let $x \in L_{n}\left(G_{0}\right)$. By definition,

$$
\Gamma^{0}(x)=\{x\} \subset L_{n}\left(G_{0}\right) .
$$

The set $\Gamma^{0}(x)$ is said to be trivial if $x \in \operatorname{Image}\left\{L_{n}\left(\rho_{0}\right) \rightarrow L_{n}\left(G_{0}\right)\right\}$. Let a set

$$
\Gamma^{j}(x) \subset L_{n-j}\left(G_{j}\right) \quad(0 \leq j \leq k-1)
$$

be defined. For $j \geq 1$, it is called trivial if $0 \in \Gamma^{j}(x)$.

If $\Gamma^{j}(x)(0 \leq j \leq k-1)$ is defined and nontrivial, then the $(j+1)$-th Browder-Livesay invariant with respect filtration (1.6) is the set

$$
\partial_{j}\left(\Gamma^{j}(x)\right) \subset L N_{n-j-2}\left(\rho_{j-1} \rightarrow G_{j-1}\right) .
$$

The $(j+1)$-th invariant is nontrivial if $0 \notin \partial_{j}\left(\Gamma^{j}(x)\right)$.

If the $(j+1)-t h(1 \leq j \leq k-1)$ Browder-Livesay invariant is defined and trivial then the set $\Gamma^{j+1}(x)$ is defined as

$$
\Gamma^{j+1}(x) \stackrel{\text { def }}{=} \Gamma\left(\Gamma^{j}(x)\right) \stackrel{\text { def }}{=}\left\{q s^{-1}(z) \mid z \in \Gamma^{j}(x), \partial_{j}(z)=0\right\} \subset L_{n-j-1}\left(G_{j+1}\right) .
$$


Theorem 1. (See [5].) Let $x \in L_{n}\left(G_{0}\right)$ be an element with a nontrivial $j$-th Browder-Livesay invariant for some $j \geq 1$ relative to a Browder-Livesay filtration $\mathcal{X}$ of the manifold $X$. Then the element $x$ cannot be realized by a normal map of closed manifolds.

Note that the necessary condition for nontriviality of the $j$-th Browder-Livesay invariant of $x \in L_{n}\left(G_{0}\right)(1 \leq j)$ is nontriviality of $\Gamma^{j-1}(x)$.

Taking in (1.2) a 3 -dimensional manifold $M$ with $\pi_{1}(M) \cong Q$ we obtain a surgery obstruction $\sigma(f) \in L_{1}(Q)$ (see [1]). The proof of nontriviality $\sigma(f)$ in [1] is based on a consideration of the Browder-Livesay filtration

$$
X_{3} \subset X_{2} \subset X_{1} \subset X_{0}=X, \quad\left(\operatorname{dim} X_{3} \geq 5\right)
$$

with $\pi_{1}(X)=Q, \pi_{1}\left(X_{1}\right)=Q^{+,-}, \pi_{1}\left(X_{2}\right)=Q^{-,-}$, and $\pi_{1}\left(X_{3}\right)=Q$. In particular, it follows from $[1]$, that $\Gamma^{3}(\sigma(f))=0$ for filtration in (1.8).

In [5] was introduced the notion of a type of any element $x \in L_{n}(\pi)$ and it was proved that the elements of the first and the second type cannot be realized by normal maps of closed manifolds. For any element $x$ of the second type there exists an "infinite" Browder-Livesay filtration for which $\Gamma^{k}(x)$ is nontrivial for all $k \geq 0$. Every element $x \in L_{n}(\pi)$ which lays in the subgroup generated by surgery obstructions of normal maps of closed manifolds has the third type. And if an element $x$ has the third type, then for any Browder-Livesay filtration there exists a finite $k$, such that $\Gamma^{k}(x)$ is trivial. In all known to the authors cases $\Gamma^{3}(x)$ is trivial for the elements of the third type.

In the present paper, we prove that $\Gamma^{3}(\sigma(f))$ is trivial for any Browder-Livesay filtration of a manifold $X^{4 k+1}$ with $\pi_{1}(X) \cong Q$, where $\sigma(f) \in L_{1}(Q)$. We compute splitting obstruction groups $L N_{*}$ and surgery obstruction groups $L P_{*}$ for various inclusions $\rho \rightarrow Q$ of index 2 , describe natural maps in the braids of exact sequences in (1.5), and make more precise several results about surgery obstruction groups of the group $Q$.

We use the surgery and splitting obstruction groups equipped with decoration "s" and we do not mention this in designations.

\section{Browder-Livesay filtrations for a manifold $X^{4 k+1}$ with $\pi_{1}(X)=Q$.}

Let $Q$ be the quaternion group from (1.1). We define an orientation homomorphism $w: Q \rightarrow$ $\{ \pm 1\}$ on generators $x$ and $y$ and denote it by superscripts in the following way:

$$
\begin{gathered}
w(x)=w(y)=1, \quad(Q, w)=Q^{+} \\
w(x)=1, w(y)=-1, \quad(Q, w)=Q^{+,-} \\
w(x)=-1, w(y)=1, \quad(Q, w)=Q^{-,+}
\end{gathered}
$$

and

$$
w(x)=-1, w(y)=-1, \quad(Q, w)=Q^{-,-} \quad(\text { in this case } w(x y)=1) .
$$

Let $\rho=\mathbb{Z} / 4$ be the cyclic group with generator $t$. There are only two homomorphisms of orientation on the group $\rho$ (homomorphisms $\mathbb{Z} / 4$ into the group \pm 1 ), and we shall write $\rho^{+}=\mathbb{Z} / 4^{+}$ in the case of trivial orientation and $\rho^{-}=\mathbb{Z} / 4^{-}$in the opposite case. The group $Q$ has only three different subgroups of index 2 generated by $x, y$, and $x y$. All these subgroups are isomorphic to $\mathbb{Z} / 4$.

Consider the Browder-Livesay filtration

$$
X_{3} \subset X_{2} \subset X_{1} \subset X_{0}=X, \quad\left(\operatorname{dim} X_{3} \geq 5\right)
$$

of a manifold $X$ with $\pi_{1}(X) \cong Q$. 
Filtration in (2.1) yields a commutative diagram

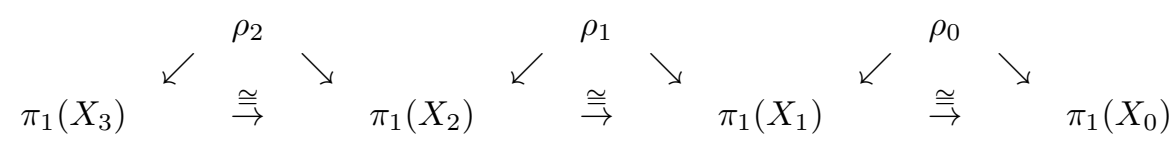

where every triangle corresponds to the square $F_{i}$, since we have isomorphisms of oriented groups $\rho_{i}=\pi_{1}\left(\partial U_{i+1}\right) \cong \pi_{1}\left(X_{i} \backslash X_{i+1}\right)$ for $0 \leq i \leq 2$.

The diagram in (2.2) is commutative as a diagram of groups, and the skew maps are inclusions of index 2 preserving the orientations. The horizontal maps in (2.2) preserve the orientation on the images of skew maps and reverse the orientation outside these images. Two diagrams as in (2.2) are isomorphic if there exists an isomorphism between them preserving orientations.

Theorem 2. Let $X^{n}, n \geq 8$, be a closed topological manifold with $\pi_{1}(X) \cong Q$. Then the diagram (2.2) is isomorphic to one of the following diagrams

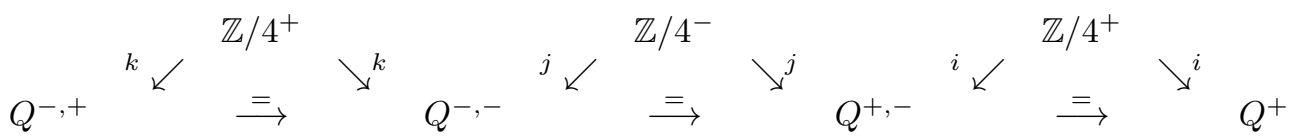

where skew homomorphisms are given on the generator $t$ by the maps $i(t)=x, j(t)=y, k(t)=x y$;

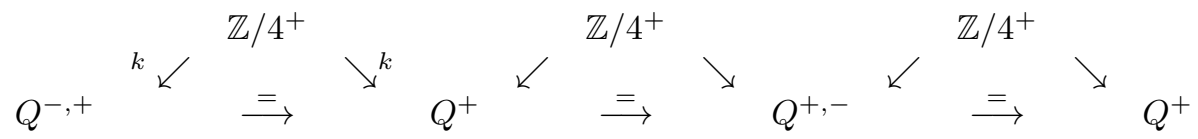

where $k: \mathbb{Z} / 4 \rightarrow Q$ is given on the generator $t$ by the map $t \rightarrow y$ and other skew homomorphisms are given on the generator $t$ by the map $t \rightarrow x$;

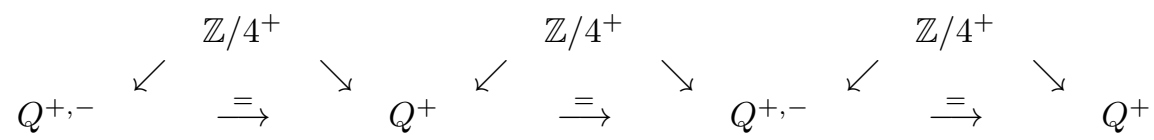

where all homomorphisms $\mathbb{Z} / 4 \rightarrow Q$ are given on the generator $t$ by the map $t \rightarrow x$;

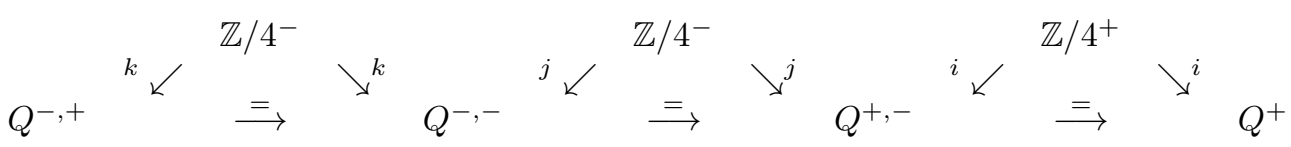

where skew homomorphisms are given on the generator $t$ by the maps $i(t)=x, j(t)=y, k(t)=y$;

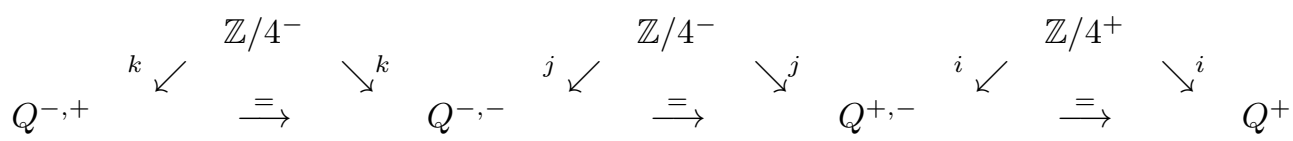

where skew homomorphisms are given on the generator $t$ by the maps $i(t)=x, j(t)=y, k(t)=x$.

For any diagram from the list (2.3)-(2.7) there exists a Browder-Livesay filtration (2.1) of the manifold $X^{n}$ which gives this diagram.

Proof. There exist isomorphisms of the oriented groups

$$
Q^{+,-} \cong Q^{-,+} \cong Q^{-,-}
$$


since there exist automorphisms of the group $Q$ permuting $x, y$, and $x y$. We have only three index 2 subgroups of $Q$ generated by $x, y$, and $x y$, which are isomorphic to $\mathbb{Z} / 4$. From this follows that all skew maps in (2.3) are inclusions of $\mathbb{Z} / 4$ to $Q$ preserving orientation. Now a consideration of various cases which agree with orientation conditions on the horizontal maps gives the first statement of the proposition. Consider the characteristic map $\phi: X^{n} \rightarrow R P^{N}$ ( $N$ sufficiently large) of the subgroup $i\left(\mathbb{Z} / 4^{+}\right)=i\left(\rho^{+}\right) \subset Q^{+}$generated by $x$ such that $\operatorname{Ker} \phi=\rho^{+}$. The transversal preimage of $R P^{N-1}$ gives a submanifold $X_{1} \subset X$ such that $X_{1} \subset X$ is a Browder-Livesay pair with the commutative triangle

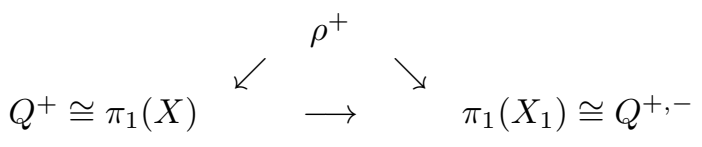

of groups (see [3] and [5]). Iteration of this construction gives the second statement of the proposition.

Every diagram from Proposition 1 induces diagram (1.7). The surgery obstruction groups fitting into the diagram (1.7) are well known [10]:

$$
\begin{array}{ccccc}
i= & 0 & 1 & 2 & 3 \\
L_{i}\left(\mathbb{Z} / 4^{+}\right) \cong & \mathbb{Z}^{3} & 0 & \mathbb{Z} \oplus \mathbb{Z} / 2 & \mathbb{Z} / 2 \\
L_{i}\left(\mathbb{Z} / 4^{-}\right) \cong & 0 & 0 & \mathbb{Z} / 2 & (\mathbb{Z} / 2)^{2} \\
L_{i}(Q) \cong & (\mathbb{Z})^{5} & |4| & \mathbb{Z} / 2 & (\mathbb{Z} / 2)^{2} \\
L_{i}\left(Q^{+,-}\right) \cong & |4| & 0 & \mathbb{Z} \oplus \mathbb{Z} / 2 & |4|,
\end{array}
$$

where $|4|$ denotes a two-group of order 4 .

Let $n=4 k+1$ and $\pi_{1}\left(X^{n}\right) \cong Q$. In this case commutative diagram (2.3) induces a diagram $($ see $[1])$

$$
\begin{aligned}
& 0=L_{1}\left(\mathbb{Z} / 4^{+}\right) \quad \stackrel{0}{\rightarrow} \quad L_{1}\left(Q^{+}\right)
\end{aligned}
$$

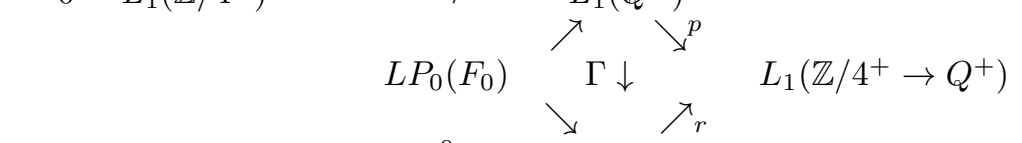

$$
\begin{aligned}
& 0=L N_{0}\left(\mathbb{Z} / 4^{+} \rightarrow Q^{+}\right) \quad \stackrel{0}{\rightarrow} \quad \begin{array}{c}
L_{0}\left(Q^{+,-}\right) \\
\end{array} \\
& 0=L_{0}\left(\mathbb{Z} / 4^{-}\right) \quad \stackrel{0}{\rightarrow} \quad L_{0}\left(Q^{+,-}\right)
\end{aligned}
$$

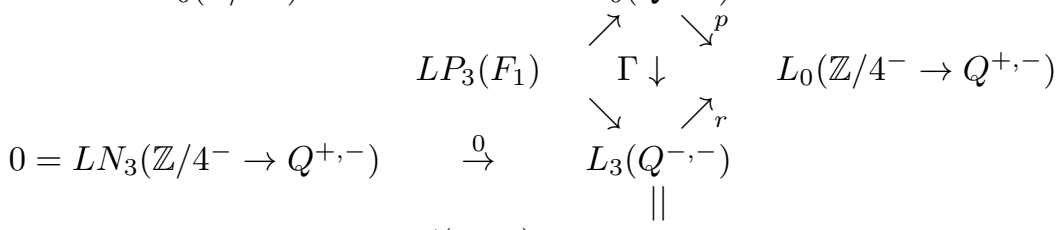

$$
\begin{aligned}
& \mathbb{Z} / 2 \cong L_{3}\left(\mathbb{Z} / 4^{+}\right) \quad \stackrel{\phi(\text { mono })}{\longrightarrow} \quad L_{3}\left(Q^{-,-}\right) \\
& \begin{array}{ccc}
\longrightarrow & L_{3}\left(Q^{-,-}\right) \\
& \nearrow & \searrow \\
L P_{2}\left(F_{2}\right) & \Gamma \downarrow & L_{3}\left(\mathbb{Z} / 4^{+} \rightarrow Q^{-,-}\right)
\end{array} \\
& L N_{2}\left(\mathbb{Z} / 4^{+} \rightarrow Q^{-,-}\right) \quad \rightarrow \quad L_{2}\left(Q^{+,+}\right) \text {. }
\end{aligned}
$$

where the groups $L N_{0}\left(\mathbb{Z} / 4^{+} \rightarrow Q^{+}\right)=0$ and $L N_{3}\left(\mathbb{Z} / 4^{-} \rightarrow Q^{+,-}\right)=0$ were computed in [1]. 
All maps $p$ and $r$ in (2.8) are monomorphisms, and the surgery obstruction $\sigma(f) \in L_{1}\left(Q^{+}\right)$has the following properties [1]:

$$
\begin{aligned}
& p(\sigma(f))=r(\sigma(g)) \text { for some element } \sigma(g) \in L_{0}\left(Q^{+,-}\right), \\
& p(\sigma(g))=r(\sigma(h)) \text { for some element } \sigma(h) \in L_{3}\left(Q^{-,-}\right),
\end{aligned}
$$

and

$$
\sigma(h)=\phi(a)
$$

where $a \in L_{3}\left(\mathbb{Z} / 4^{+}\right) \cong \mathbb{Z} / 2$ is the nontrivial element.

Note, that the results of [1] immediately imply that $\Gamma^{3}(\sigma(f))=0$ for the diagram (2.3).

\section{Computing $\Gamma^{i}$ for Browder-Livesay filtrations.}

In this section we prove the following result: let $X^{4 k+1}(4 k+1 \geq 9)$ be a manifold with $\pi_{1}(X) \cong Q$. Consider the Browder-Livesay filtration of $X$ with a diagram of inclusion (2.3) (2.7). Then $\Gamma^{3}(\sigma(f))=0$ for the Cappell-Shaneson surgery obstruction $\sigma(f) \in L_{1}\left(Q^{+}\right)$.

The statement of the theorem for diagram (2.3) follows from diagram (2.8) and results of [1]. We shall give the statement of the theorem for other diagrams $(2.4)-(2.7)$ in Theorems 3 and 4 of this section.

We introduce the following notations (see [10]):

$$
\begin{aligned}
& T^{-,-}=\mathbb{Z}\left[Q^{-,-}\right], \quad \hat{T}_{2}^{-,-}=\hat{\mathbb{Z}}_{2}\left[Q^{-,-}\right], \\
& R^{+}=\mathbb{Z}\left[\mathbb{Z} / 4^{+}\right], \quad \hat{R}_{2}^{+}=\hat{\mathbb{Z}}_{2}\left[\mathbb{Z} / 4^{+}\right],
\end{aligned}
$$

and similarly for other orientations. Denote by $L_{n}^{r e l}\left(T^{-,-}\right)$the relative groups $L_{n}\left(T^{-,-} \rightarrow T_{2}^{-,-}\right)$ fitting into the relative exact sequence

$$
\rightarrow L_{n}\left(T^{-,-}\right) \rightarrow L_{n}\left(\hat{T}_{2}^{-,-}\right) \rightarrow L_{n}\left(T^{-,-} \rightarrow T_{2}^{-,-}\right) \rightarrow L_{n-1}\left(T^{-,-}\right) \rightarrow
$$

and similarly for other group rings and orientations. ¿From the isomorphisms $Q^{+,-} \cong Q^{-,+} \cong$ $Q^{-,-}$which were described in Section 2, we obtain isomorphisms

$$
\begin{aligned}
& T^{-,-} \cong T^{-,+} \cong T^{+,-}, \\
& \hat{T}_{2}^{-,-} \cong \hat{T}_{2}^{+,-} \cong \hat{T}_{2}^{-,+}
\end{aligned}
$$

and isomorphisms

$$
L_{n}^{Y}\left(T^{-,-} \rightarrow T_{2}^{-,-}\right) \cong L_{n}^{Y}\left(T^{+,-} \rightarrow T_{2}^{+,-}\right) \cong L_{n}^{Y}\left(T^{-,+} \rightarrow T_{2}^{-,+}\right) .
$$

We shall consider the $L$-groups with decorations "prime". For the case of a group ring $\mathbb{Z}[\pi]$ these $L$-groups coincide with surgery obstruction groups. We have isomorphisms (see [1] and [10])

$$
L N_{n}\left(\mathbb{Z} / 4^{+} \rightarrow Q^{+,+}\right) \cong L_{n}\left(\mathbb{Z}[\mathbb{Z} / 4], \mathrm{Id},-t^{2}\right)=L_{n}(B)
$$

and the relative exact sequence

$$
\rightarrow L_{n}\left(\mathbb{Z}[\mathbb{Z} / 4], \mathrm{Id},-t^{2}\right) \rightarrow L_{n}\left(\hat{\mathbb{Z}}_{2}[\mathbb{Z} / 4], \mathrm{Id},-t^{2}\right) \rightarrow L_{0}^{r e l}\left(\mathbb{Z}[\mathbb{Z} / 4], \mathrm{Id},-t^{2}\right) \rightarrow
$$

Denote

$$
L_{n}(B)=L_{n}\left(\mathbb{Z}[\mathbb{Z} / 4], \mathrm{Id},-t^{2}\right)
$$

and

$$
L_{n}\left(\hat{B}_{2}\right)=L_{n}\left(\hat{\mathbb{Z}}_{2}[\mathbb{Z} / 4], \mathrm{Id},-t^{2}\right)
$$


By [1], we have

$$
L N_{n}\left(\mathbb{Z} / 4^{-} \stackrel{j}{\rightarrow} Q^{-,-}\right) \cong L_{n}\left(\mathbb{Z}[\mathbb{Z} / 4], D, \pm t^{2}\right)=L_{n}(A)
$$

where $D(t)=-t$. In particular, we have an isomorphism

$$
L N_{n}\left(\mathbb{Z} / 4^{-} \stackrel{j}{\rightarrow} Q^{-,-}\right) \cong L N_{n+2}\left(\mathbb{Z} / 4^{-} \stackrel{j}{\rightarrow} Q^{-,-}\right) .
$$

Denote

$$
L_{n}(A)=L_{n}\left(\mathbb{Z}[\mathbb{Z} / 4], D, \pm t^{2}\right)=
$$

and

$$
L_{n}\left(\hat{A}_{2}\right)=L_{n}\left(\hat{\mathbb{Z}}_{2}[\mathbb{Z} / 4], D, \pm t^{2}\right) .
$$

These groups fit into the relative exact sequence

$$
\rightarrow L_{n}\left(\mathbb{Z}[\mathbb{Z} / 4], D, \pm t^{2}\right) \rightarrow L_{n}\left(\hat{\mathbb{Z}}_{2}[\mathbb{Z} / 4], D, \pm t^{2}\right) \rightarrow L_{n}^{r e l}\left(\mathbb{Z}[\mathbb{Z} / 4], D, \pm t^{2}\right) \rightarrow
$$

Braid of exact sequences (1.5) also exists for relative groups (see [6] and [10]). For the inclusions $\mathbb{Z} / 4^{ \pm} \rightarrow Q^{+, \pm}$, all relative groups and all groups for the the group ring over the ring $\hat{\mathbb{Z}}_{2}$ are known [10]. In particular, we have

$$
\begin{array}{ccccccc}
n & & 0 & 1 & 2 & 3 \\
L_{n}^{r e l}\left(R^{+}\right)= & L_{n}^{Y}\left(R^{+} \rightarrow R_{2}^{+}\right) & \cong & 0 & \mathbb{Z}^{3} \oplus(\mathbb{Z} / 2)^{2} & 0 & \mathbb{Z} \\
L_{n}^{r e l}\left(T^{+}\right)= & L_{n}^{Y}\left(T^{+} \rightarrow T_{2}^{+}\right) & \cong & 0 & \mathbb{Z}^{5} \oplus(\mathbb{Z} / 2)^{4} & \mathbb{Z} / 2 & (\mathbb{Z} / 2)^{2} \\
L_{n}^{r e l}(B)= & L_{n}^{Y}\left(B \rightarrow \hat{B}_{2}\right) & \cong & \mathbb{Z} / 2 & (\mathbb{Z} / 2)^{2} & 0 & (\mathbb{Z})^{2} \oplus(\mathbb{Z} / 2)^{2} \\
L_{n}^{r e l}\left(R^{-}\right)= & L_{n}^{Y}\left(R^{-} \rightarrow R_{2}^{-}\right) & \cong & \mathbb{Z} / 2 & \mathbb{Z} / 2 \oplus \mathbb{Z} / 2 & 0 & 0 \\
L_{n}^{r e l}\left(T^{-}\right)= & L_{n}^{Y}\left(T^{-,-} \rightarrow T_{2}^{-,-}\right) & \cong & \mathbb{Z} / 2 & \mathbb{Z} / 2 \oplus \mathbb{Z} / 2 & 0 & \mathbb{Z} \\
L_{n}^{\text {rel }}(A)= & L_{n}^{Y}\left(A \rightarrow \hat{A}_{2}\right) & \cong & 0 & \mathbb{Z} & 0 & \mathbb{Z}
\end{array}
$$

Consider the diagram

$$
\begin{aligned}
& 0=L_{1}\left(\mathbb{Z} / 4^{+}\right) \\
& \stackrel{0}{\rightarrow} \quad L_{1}\left(Q^{+}\right) \\
& s \nearrow \cong \\
& \downarrow \downarrow L_{1}\left(\mathbb{Z} / 4^{+} \rightarrow Q^{+}\right) \\
& 0=L N_{0}\left(\mathbb{Z} / 4^{+} \rightarrow Q^{+}\right) \quad \stackrel{0}{\rightarrow} \quad \stackrel{L_{0}\left(Q^{+,-}\right)}{\|} \\
& L_{0}\left(\mathbb{Z} / 4^{+}\right) \quad \stackrel{I m=\mathbb{Z} / 2}{\rightarrow} \quad L_{0}\left(Q^{+,-}\right) \\
& s \nearrow_{\Gamma \downarrow} \searrow \quad L_{0}\left(\mathbb{Z} / 4^{+} \rightarrow Q^{+,-}\right)=\mathbb{Z} / 2 \\
& \mathbb{Z} / 2=L N_{3}\left(\mathbb{Z} / 4^{+} \rightarrow Q^{+,-}\right) \quad \stackrel{\text { mono }}{\rightarrow} \quad \begin{array}{c}
q \searrow \\
L_{3}\left(Q^{+,+}\right)=\left({ }^{\mathbb{Z}} / 2\right)^{2}
\end{array} \\
& L_{3}\left(\mathbb{Z} / 4^{+}\right) \quad \stackrel{\text { mono }}{\rightarrow} \quad L_{3}\left(Q^{+,+}\right) \\
& s \nearrow_{\Gamma \downarrow}^{L_{3}\left(Q^{+,+}\right)} \\
& q \searrow \quad \nearrow \\
& L N_{2}\left(\mathbb{Z} / 4^{+} \rightarrow Q^{+,+}\right) \quad \rightarrow \quad L_{2}\left(Q^{+,-}\right) .
\end{aligned}
$$

which is defined by diagram (2.4). Diagram (3.2) is similar to diagram (2.8). In the group $L_{0}\left(Q^{+,-}\right)$ lies the element $\sigma(g)$ which is the image of the element $\sigma(f) \in L_{1}\left(Q^{+}\right)$under the upper map $\Gamma$ in (3.1) as follows from [1]. 
Theorem 3. In diagram (3.2), the element $\sigma(g) \in L_{0}\left(Q^{+,-}\right)$lies in the image of the map

$$
L_{0}\left(\mathbb{Z} / 4^{+}\right) \longrightarrow L_{0}\left(Q^{+,-}\right),
$$

and hence $\Gamma^{2}(\sigma(f))=0$. From this it immediately follows that $\Gamma^{2}(\sigma(f))=0$ for diagrams (2.4) and (2.5).

Proof. Follows by Lemma 1 and Lemma 2.

Lemma 1. Let a be the nontrivial element of the relative group $L_{0}\left(T^{-,-} \rightarrow \hat{T}_{2}^{-,-}\right)=\mathbb{Z} / 2$ and

$$
\partial: L_{0}\left(T^{-,-} \rightarrow \hat{T}_{2}^{-,-}\right) \rightarrow L_{3}\left(T^{-,-}\right)
$$

be a map from the corresponding relative exact sequence. Then

$$
\partial(a)=\sigma(h)=\phi(x), \text { where } \quad x \in L_{3}\left(\mathbb{Z} / 4^{+}\right), \quad x \neq 0 .
$$

Proof. Consider the commutative diagram

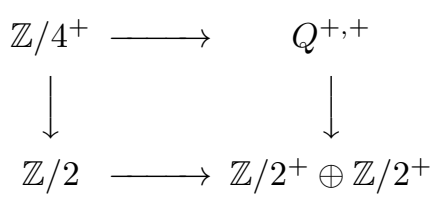

in which vertical maps are natural projections. By [10], the vertical maps in (3.3) induce a retraction of the braid of exact sequences of relative groups for the top inclusion in (3.3) to the corresponding braid of exact sequences of relative groups for the bottom inclusion in (3.3).

As the kernel of this retraction we obtain a braid of exact sequences with known groups and easily computed maps. All relative groups for the inclusion $\mathbb{Z} / 2^{+} \rightarrow \mathbb{Z} / 2^{+} \oplus \mathbb{Z} / 2^{+}$are known from [10]:

$$
\begin{array}{cccccc}
n & = & 0 & 1 & 2 & 3 \\
L_{n}\left(\mathbb{Z}[\mathbb{Z} / 2] \rightarrow \hat{\mathbb{Z}}_{2}[\mathbb{Z} / 2]\right. & \cong & 0 & (\mathbb{Z} \oplus \mathbb{Z} / 2)^{2} & 0 & 0 \\
L_{n}\left(\mathbb{Z}\left[(\mathbb{Z} / 2)^{2}\right] \rightarrow \hat{\mathbb{Z}}_{2}\left[(\mathbb{Z} / 2)^{2}\right]\right. & \cong & 0 & (\mathbb{Z} \oplus \mathbb{Z} / 2)^{4} & 0 & 0 .
\end{array}
$$

All relative groups for the inclusion $\mathbb{Z} / 4^{+} \rightarrow Q^{+,+}$were given in (3.1) (see [10]). From this we conclude that the map

$$
\mathbb{Z} / 2=L_{0}^{r e l}\left(\mathbb{Z}[\mathbb{Z} / 4], \mathrm{Id},-t^{2}\right) \stackrel{\xi}{\longrightarrow} L_{0}^{r e l}\left(T^{+,-}\right)=\mathbb{Z} / 2
$$

of relative groups is an isomorphism. The map $\xi$ from (3.4) lies in the commutative diagram

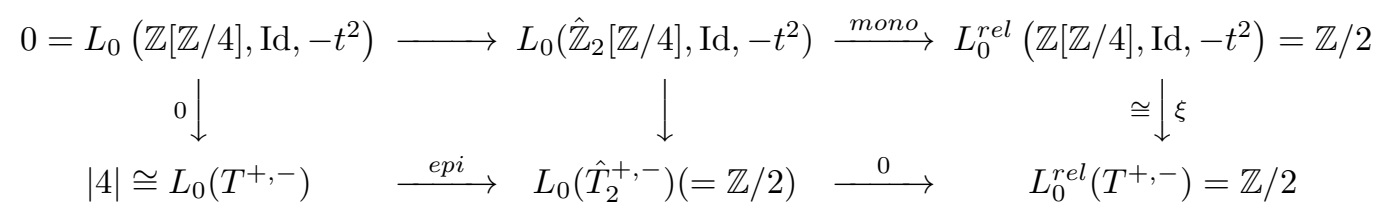

in which top row is a part of relative exact sequence for an inclusion $B \rightarrow \hat{B}_{2}$, the left group in top row is trivial by [1], the bottom row is the part of the relative exact sequence for an inclusion $T^{+,-} \rightarrow \hat{T}_{2}^{+,-}$, and the group and the maps in the bottom row were described in [10]. Since the map $\xi$ is an isomorphism, we obtain from commutativity of diagram (3.5) that

$$
L_{0}\left(\hat{\mathbb{Z}}_{2}[\mathbb{Z} / 4], \mathrm{Id},-t^{2}\right)=0
$$


Consider a part

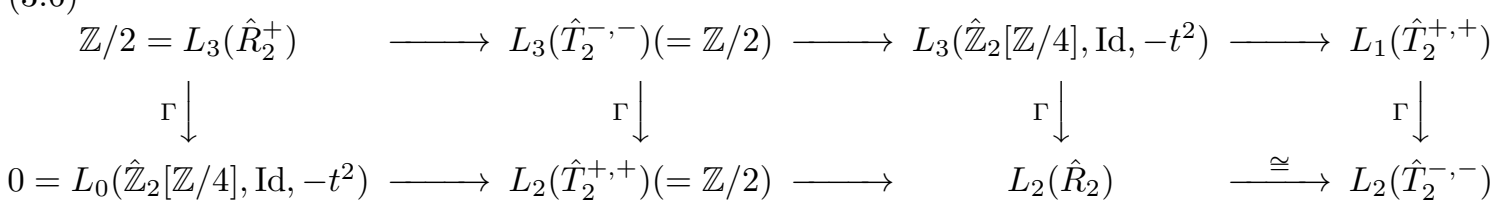

of the two-row diagram for the inclusion $\hat{R}_{2}^{+} \rightarrow \hat{T}_{2}^{-,-}$which corresponds to the inclusion $\mathbb{Z} / 4^{+} \rightarrow$ $Q^{-,-}$given by the map $t \rightarrow x y$. The rows of diagram (3.6) are chain complexes with isomorphic homology groups. The right bottom horizontal map is an isomorphism $\mathbb{Z} / 2 \rightarrow \mathbb{Z} / 2$ (Arf-invariant), hence the homology group in the member $L_{2}\left(\hat{T}_{2}^{+,+}\right)$is $\mathbb{Z} / 2$. Hence in top row the homology group in the member $L_{3}\left(\hat{T}_{2}^{-,-}\right)$is $\mathbb{Z} / 2$ and hence the induced by the inclusion $\mathbb{Z} / 4^{+} \rightarrow Q^{-,-}(t \rightarrow x y)$ map

$$
\alpha: \mathbb{Z} / 2=L_{3}\left(\hat{R}_{2}^{+}\right) \rightarrow L_{3}\left(\hat{T}_{2}^{-,-}\right)=\mathbb{Z} / 2
$$

is trivial.

The inclusion $\mathbb{Z} / 4^{+} \rightarrow Q^{-,-}(t \rightarrow x y)$ induces the map of relative exact sequences, and we obtain the commutative diagram

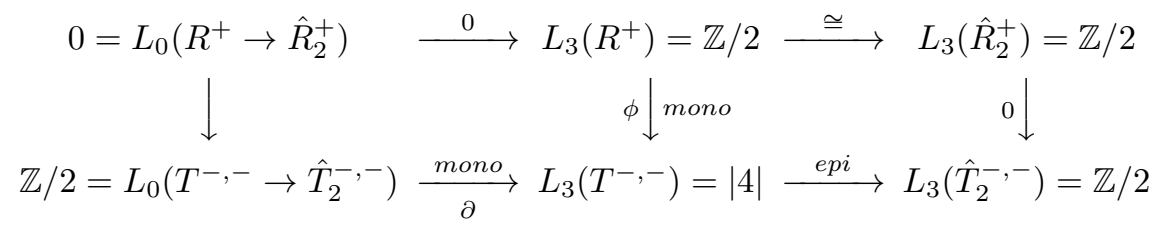

where the right hand vertical map is trivial as we have proved. Now the statement of Lemma 1 follows from diagram (3.8).

For the inclusion $\mathbb{Z} / 4^{-} \rightarrow Q^{+,-}(t \rightarrow y)$ we consider the following part of diagram (3.2)

$$
\begin{array}{lll}
L_{0}\left(Q^{+,-}\right) & & \\
& \searrow^{p} & \\
& & \\
& & \\
L_{3}\left(Q^{-,-}\right) & & \left.\mathbb{Z} / 4^{-} \rightarrow Q^{+,-}\right) \\
& &
\end{array}
$$

where $\sigma(g) \in L_{0}\left(Q^{+,-}\right)$and $\sigma(h) \in L_{3}\left(Q^{-,-}\right)$. We have a natural map of the corresponding diagram of relative groups to diagram (3.2) and hence a map of a diagram of relative groups to diagram (3.9). Thus we obtain the commutative diagram

$$
\begin{aligned}
& 0 \neq b \in \mathbb{Z} / 2 \oplus \mathbb{Z} / 2=L_{1}\left(T^{+,-} \rightarrow \hat{T}_{2}^{+,-}\right) \stackrel{I m=\mathbb{Z} / 2}{\underset{\delta}{\longrightarrow}} L_{0}\left(Q^{+,-}\right) \text {э } \sigma(g) \\
& \text { epi } \quad p \downarrow \text { mono } \\
& \mathbb{Z} / 2=L_{1}\left(\begin{array}{ccc}
R^{-} & \rightarrow & \hat{R}_{2}^{-} \\
\downarrow & & \downarrow \\
T^{+,-} & \rightarrow & \hat{T}_{2}^{+,-}
\end{array}\right) \quad \stackrel{\text { mono }}{\longrightarrow} L_{0}\left(\mathbb{Z} / 4^{-} \rightarrow Q^{+,-}\right) \\
& \cong \uparrow \quad r \uparrow \text { mono } \\
& 0 \neq a \in \mathbb{Z} / 2=L_{0}\left(T^{-,-} \rightarrow \hat{T}_{2}^{-,-}\right) \quad \stackrel{\text { mono }}{\longrightarrow} L_{3}\left(Q^{-,-}\right) \text {э } \sigma(h)
\end{aligned}
$$


in which the maps $p$ and $r$ are monomorphisms by [1]. The middle horizontal map in (3.10) is a monomorphism since the diagonal map

$$
L_{0}\left(T^{-,-} \rightarrow \hat{T}_{2}^{-,-}\right) \rightarrow L_{0}\left(\mathbb{Z} / 4^{-} \rightarrow Q^{+,-}\right)
$$

from bottom square is a monomorphism as follows from Lemma 1. The left hand upper vertical map in (3.10) is an epimorphism by [10]. Hence from commutativity of (3.10) we obtain that there exists an element

$$
b \in L_{1}\left(T^{+,-} \rightarrow \hat{T}_{2}^{+,-}\right)
$$

such that $\delta(b)=\sigma(g) \in L_{0}\left(Q^{+,-}\right)$.

Consider the map of relative exact sequence of the inclusion $R^{+} \rightarrow \hat{R}_{2}^{+}$to relative exact sequence of the inclusion $T^{+,-} \rightarrow \hat{T}_{2}^{+,-}$which is induced by the inclusion $\mathbb{Z} / 4^{+} \rightarrow Q^{+,-}$. We can write down the commutative diagram

$$
\begin{array}{ccc}
\mathbb{Z}^{3} \oplus(\mathbb{Z} / 2)^{3}=L_{1}\left(R^{+} \rightarrow \hat{R}_{2}^{+}\right) & \stackrel{e p i}{\longrightarrow} & L_{0}\left(R^{+}\right)=\mathbb{Z}^{3} \\
I m=\mathbb{Z} / 2 \downarrow & i_{*} \downarrow \\
0 \neq b \in(\mathbb{Z} / 2)^{2}=L_{1}\left(T^{+,-} \rightarrow \hat{T}_{2}^{+,-}\right) & \stackrel{I m=\mathbb{Z} / 2}{\delta} & L_{0}\left(T^{+,-}\right)=|4|(\ni \sigma(g)=\delta(b))
\end{array}
$$

where the left hand vertical map follows from [10].

Lemma 2. The map

$$
\mathbb{Z}^{3}=L_{0}\left(R^{+}\right) \stackrel{i_{*}}{\rightarrow} L_{0}\left(T^{+,-}\right)
$$

in diagram (3.11) has the image $\mathbb{Z} / 2$ and hence the element $\sigma(g)$ lies in the image of this map.

Proof. Commutative diagram (3.3) induces a commutative diagram

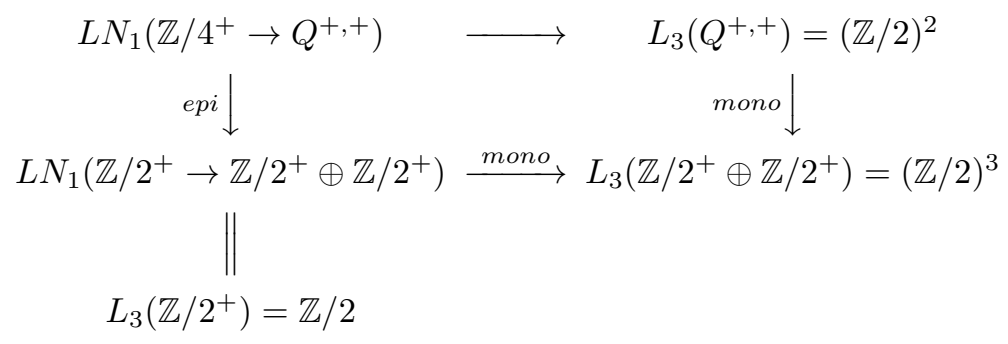

in which the right vertical map is a monomorphism by [1] and the left vertical map is an epimorphism as follows from the long exact sequence of the pull-back diagram (see [1])

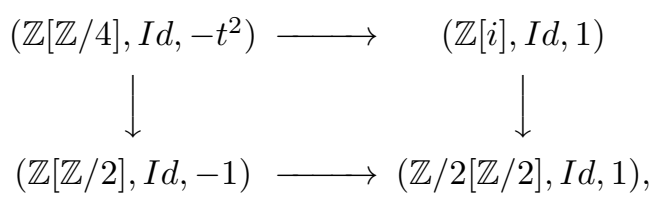

where $L N_{i}\left(\mathbb{Z} / 4^{+} \rightarrow Q^{+,+}\right)=L_{i}\left(\mathbb{Z}[\mathbb{Z} / 4], I d,-t^{2}\right)$. Thus the image of the upper horizontal map in (3.12) is $\mathbb{Z} / 2$. Now consider the following part of the two-row diagram for the inclusion $\mathbb{Z} / 4^{+} \rightarrow$ $Q^{+,-}$

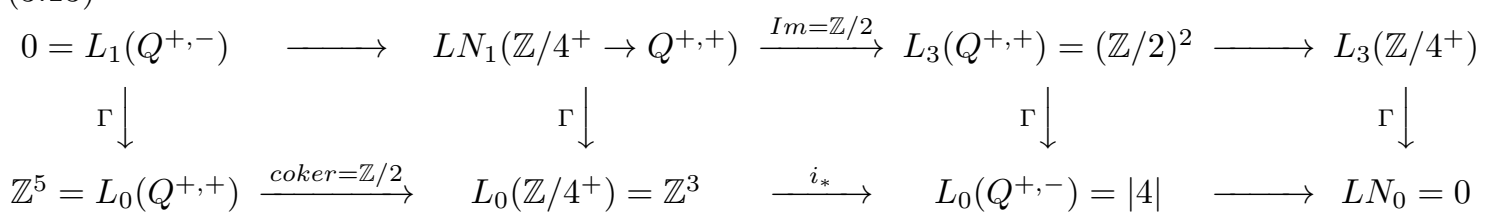


in which rows are chain complexes with isomorphic homology groups and vertical maps $\Gamma$ are isomorphisms of homologies. The map

$$
\mathbb{Z}^{5}=L_{0}\left(Q^{+,+}\right) \rightarrow L_{0}\left(\mathbb{Z} / 4^{+}\right)=\mathbb{Z}^{3}
$$

has cokernel $\mathbb{Z} / 2$ as follows from consideration of the corresponding diagram of relative groups. It follows now that image $i_{*}$ in $(3.13)$ is $\mathbb{Z} / 2$ and the lemma is proved.

Now Theorem 3 is proved.

On the base of diagram (2.6) we can write down the following commutative diagram of surgery obstruction groups

$$
\begin{aligned}
& 0=L_{1}\left(\mathbb{Z} / 4^{+}\right) \quad \stackrel{0}{\rightarrow} \quad L_{1}\left(Q^{+}\right) \\
& \nearrow \searrow p \\
& \Gamma \downarrow \quad L_{1}\left(\mathbb{Z} / 4^{+} \rightarrow Q^{+}\right) \\
& 0=L N_{0}\left(\mathbb{Z} / 4^{+} \rightarrow Q^{+}\right) \quad \stackrel{0}{\rightarrow} \quad \begin{array}{c}
L_{0}\left(Q^{+,-}\right) \\
\|
\end{array} \\
& 0=L_{0}\left(\mathbb{Z} / 4^{+}\right) \quad \stackrel{j_{*}=0}{\longrightarrow} \quad L_{0}\left(Q^{+,-}\right) \\
& \nearrow \searrow p \\
& \Gamma \downarrow \quad L_{0}\left(\mathbb{Z} / 4^{-} \rightarrow Q^{+,-}\right) \\
& L N_{3}\left(\mathbb{Z} / 4^{-} \rightarrow Q^{+,-}\right) \quad \stackrel{0}{\rightarrow} \quad L_{3}\left(Q^{-,-}\right) \\
& \mathbb{Z} / 2 \oplus \mathbb{Z} / 2 \cong L_{3}\left(\mathbb{Z} / 4^{-}\right) \quad \stackrel{j_{*}}{\longrightarrow} \quad L_{3}\left(Q^{-,-}\right) \\
& \nearrow_{\Gamma \downarrow}^{\searrow} L_{3}\left(\mathbb{Z} / 4^{-} \rightarrow Q^{-,-}\right) \\
& L N_{2}\left(\mathbb{Z} / 4^{-} \rightarrow Q^{-,-}\right) \quad \longrightarrow \quad L_{2}\left(Q^{+,-}\right)
\end{aligned}
$$

which is similar to diagram (2.8). By [1], $\sigma(g) \in L_{0}\left(Q^{+,-}\right), \sigma(h) \in L_{3}\left(Q^{-,-}\right)$, and $p(\sigma(g))=$ $r(\sigma(h))$.

Theorem 4. The map

$$
\mathbb{Z} / 2 \oplus \mathbb{Z} / 2=L_{3}\left(\mathbb{Z} / 4^{-}\right) \stackrel{j_{*}}{\longrightarrow} L_{3}\left(Q^{-,-}\right)
$$

in diagram (3.14) is an isomorphism. Hence $\Gamma^{3}(\sigma(f)) \in L_{2}\left(Q^{+,-}\right)$is trivial for diagram (3.14). From this it follows that $\Gamma^{3}(\sigma(f))=0$ for diagrams (2.6) and (2.7).

Proof. For the inclusion $j: \mathbb{Z} / 4^{-} \rightarrow Q^{+,-}$, consider a two-row diagram of relative $L$-groups, which is part of the braid of exact sequences of relative $L$-groups

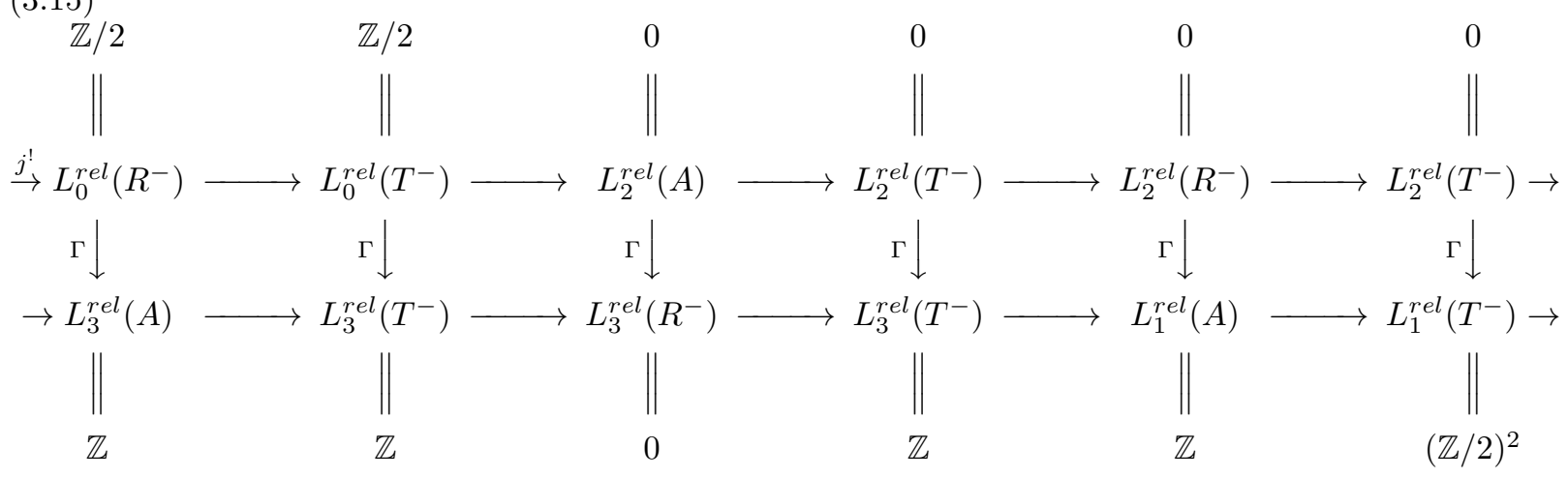




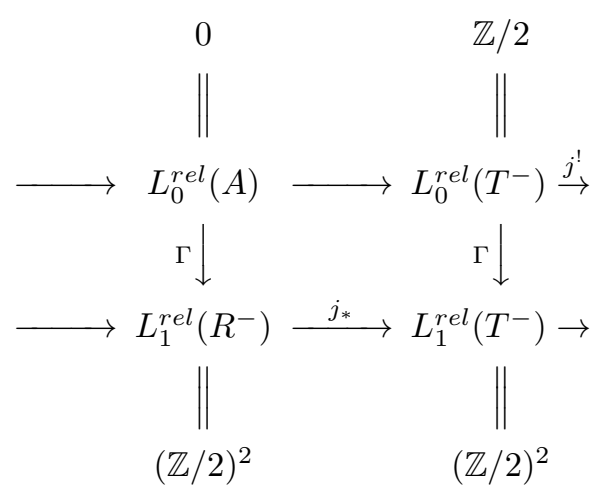

Now we shall prove Lemmas $3,4,5$, and 6 which yields a proof of Theorem 4 .

Lemma 3. In diagram (3.15), the map

$$
\mathbb{Z}=L_{3}^{r e l}(A) \rightarrow L_{3}^{r e l}\left(T^{-}\right)=\mathbb{Z}
$$

is isomorphism, and the map

$$
\mathbb{Z}=L_{3}^{r e l}\left(T^{-}\right) \rightarrow L_{1}^{r e l}(A)=\mathbb{Z}
$$

is a multiplication by 2 with coimage $\mathbb{Z} / 2$.

Proof. Suppose that the map

$$
\mathbb{Z} / 2=L_{0}^{r e l}\left(T^{-}\right) \stackrel{j^{!}}{\longrightarrow} L_{0}^{r e l}\left(R^{-}\right)=\mathbb{Z} / 2
$$

in diagram (3.15) is an isomorphism. Then, comparing homology of top and bottom rows of diagram (3.15), we obtain a contradiction. Hence the map

$$
\mathbb{Z} / 2=L_{0}^{\text {rel }}\left(R^{-}\right) \stackrel{j_{*}}{\longrightarrow} L_{0}^{\text {rel }}\left(T^{-}\right)=\mathbb{Z} / 2
$$

in top row of (3.15) is an isomorphism. From this the result follows by diagram chasing in diagram (3.15).

For an inclusion $j: \mathbb{Z} / 4^{-} \rightarrow Q^{-}$, we have a two-row diagram of $L$-groups (3.16)

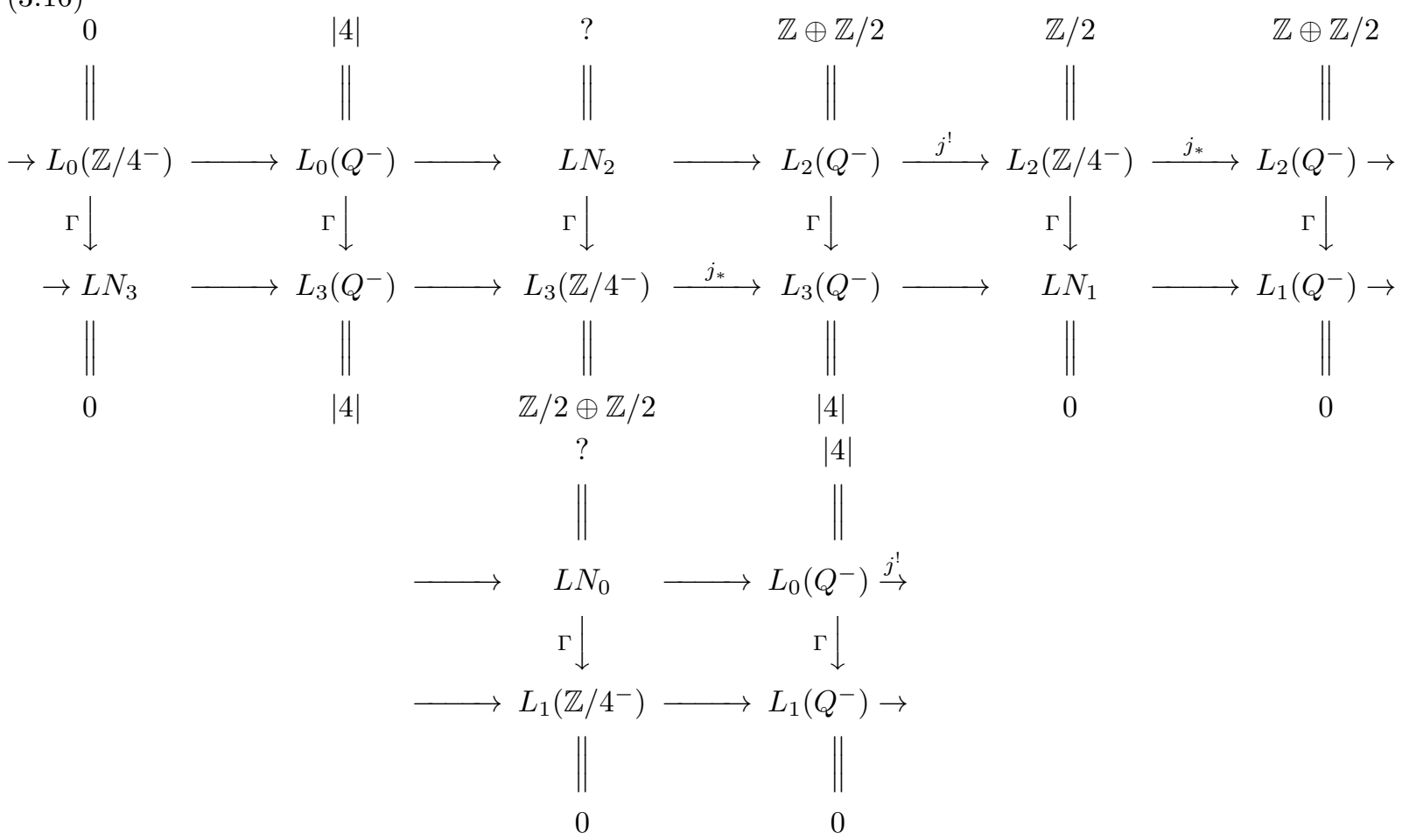


Lemma 4. In diagram (3.16), the map

$$
\mathbb{Z} / 2=L_{2}\left(\mathbb{Z} / 4^{-}\right) \stackrel{j_{*}}{\longrightarrow} L_{2}\left(Q^{-}\right)=\mathbb{Z} \oplus \mathbb{Z} / 2
$$

is a monomorphism, and hence the map

$$
L_{2}\left(Q^{-}\right) \stackrel{j^{!}}{\longrightarrow} L_{2}\left(\mathbb{Z} / 4^{-}\right)
$$

is trivial.

Proof. Consider the commutative diagram of oriented groups

$$
\begin{array}{cc}
\mathbb{Z} / 4^{-} \stackrel{j(t)=y}{\longrightarrow} \quad Q^{+,-} \\
t^{2}=1 \downarrow & x^{2}=y^{2}=1 \downarrow \\
\mathbb{Z} / 2^{-} \longrightarrow \mathbb{Z} / 2^{+} \oplus \mathbb{Z} / 2^{-} .
\end{array}
$$

Diagram (3.17) induces the following diagram of $L$-groups

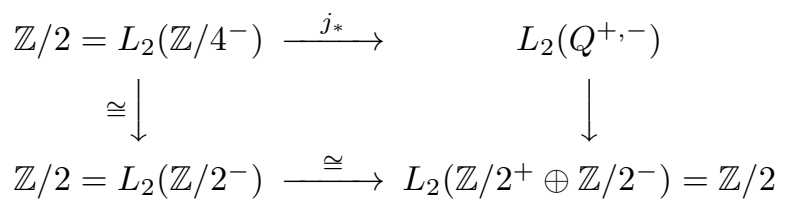

in which the left vertical map is isomorphism (Arf-invariant is preserved), and the bottom horizontal map is an isomorphism (the bottom horizontal map is an inclusion of a direct summand). Now we obtain the monomorphism of top row in (3.18). The result follows.

Lemma 5. We have isomorphisms [6]

$$
L N_{2}\left(\mathbb{Z} / 4^{-} \rightarrow Q_{3}^{-}\right) \cong L N_{0}\left(\mathbb{Z} / 4^{-} \rightarrow Q_{3}^{-}\right) \cong \mathbb{Z} \oplus \mathbb{Z} / 2
$$

and the map

$$
\mathbb{Z} \cong L_{2 n+1}^{r e l}(A) \rightarrow L_{2 n}(A) \cong L N_{2 n}\left(\mathbb{Z} / 4^{-} \rightarrow Q_{3}^{-}\right)
$$

is an inclusion of a direct summand.

Proof. Consider a commutative diagram

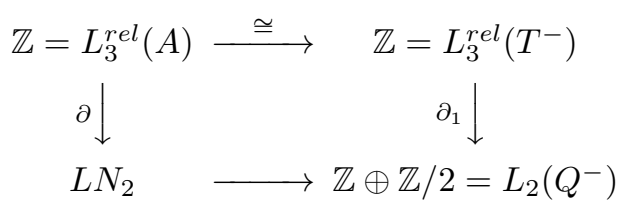

in which vertical maps fit into the corresponding relative exact sequences. The right vertical map $\partial_{1}$ in (3.19) is an inclusion of a direct summand by [10], and upper horizontal map is an isomorphism by Lemma 3. Hence the left vertical map in (3.19) is an inclusion of a direct summand. Now from diagram (3.16) we obtain the diagram 


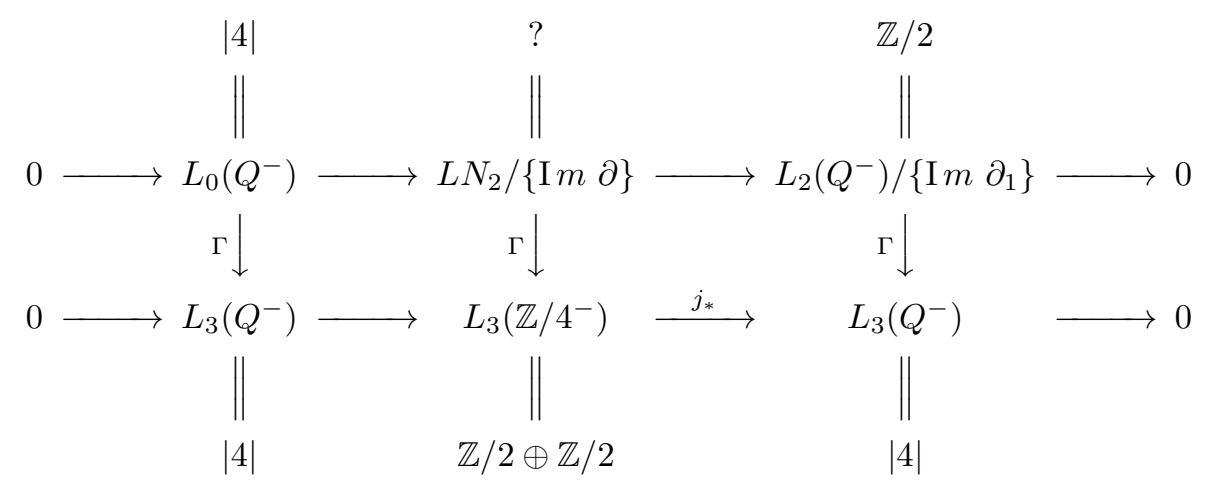

in which the homology groups in the corresponding places of upper and bottom rows are isomorphic by isomorphisms $\Gamma$. Hence we have the isomorphism

$$
L N_{2} /\{\operatorname{Im} \partial\} \cong \mathbb{Z} / 2
$$

and

$$
L N_{2} \cong \mathbb{Z} \oplus \mathbb{Z} / 2 .
$$

The isomorphism $L N_{2} \cong L N_{0}$ follows from 2-periodicity.

Lemma 6. The map

$$
\mathbb{Z} \oplus \mathbb{Z} / 2=L N_{2} \rightarrow L_{2}\left(Q^{+,-}\right)=\mathbb{Z} \oplus \mathbb{Z} / 2
$$

in diagram (3.16) is an isomorphism

Proof. Consider the commutative diagram

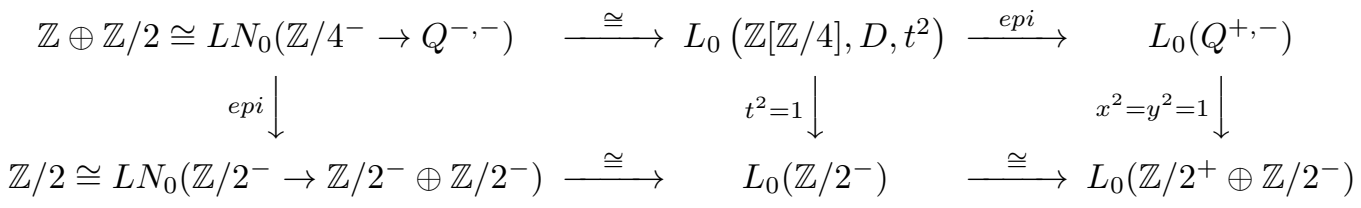

in which the top right hand horizontal map is an epimorphism as follows from (3.16). Hence the left vertical map provides an isomorphism of the torsion part $\mathbb{Z} / 2$ of the group $L N_{0}$ on the group $L_{0}\left(\mathbb{Z} / 2^{-}\right)=\mathbb{Z} / 2$ (the direct summand $\mathbb{Z}$ of the group $L N_{0}$ maps trivially since it factors acroos the corresponding map of relative groups which is trivial).

Now we can write down a commutative diagram

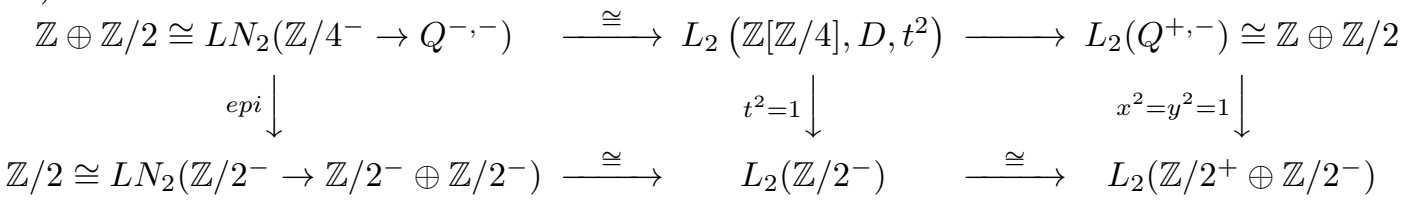

in which the left vertical map induces an isomorphism of the torsion part $\mathbb{Z} / 2$ of the group $L N_{2}$ on the group $L_{2}\left(\mathbb{Z} / 2^{-}\right)=\mathbb{Z} / 2$ by 2 -periodicity. The direct summand $\mathbb{Z}$ of the group $L N_{2}$ maps isomorphically to the direct summand $\mathbb{Z}$ of the group $L_{2}\left(Q^{+,-}\right)$as follows from Lemma 3 and Lemma 5. The diagram (3.22) provides a similar result about torsion subgroups. Lemma 6 is proved. Now Theorem 4 follows. 


\section{Braids of exact sequences.}

In this section we give explicit results about braids of exact sequences of $L_{*}$-groups for various index 2 inclusions $\rho \rightarrow Q$. The results of this section are direct corollaries of computations in Section 3 and [1], [6], and [10].

Theorem 5. For an inclusion $j: \mathbb{Z} / 4^{-} \rightarrow Q^{-}$, we have the following two-row diagram of L-groups

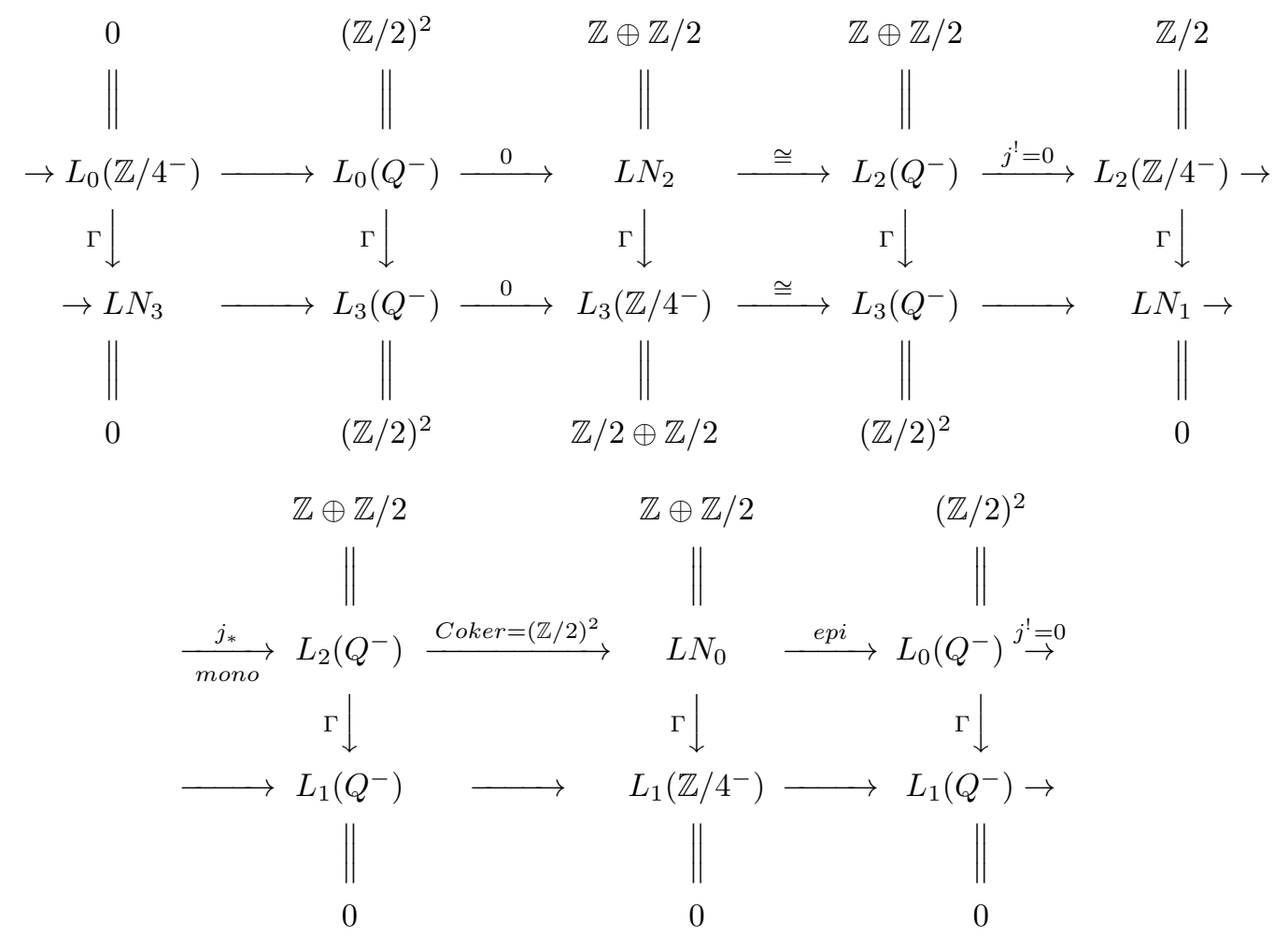

Corollary 1. There exist isomorphisms

$$
L_{0}\left(Q^{-}\right) \cong L_{3}\left(Q^{-}\right) \cong \mathbb{Z} / 2 \oplus \mathbb{Z} / 2
$$

Corollary 2. Let $Y \subset X$ be be a Browder-Livesay pair of manifolds with a push-out square of fundamental groups

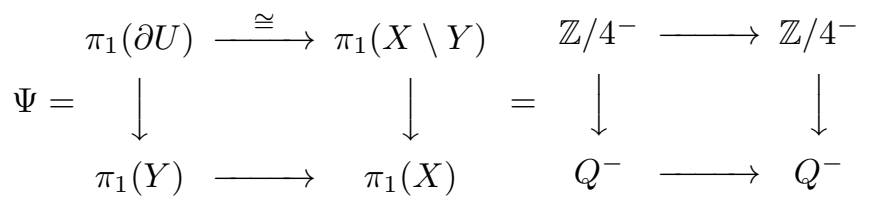

in the corresponding splitting problem. Then we have the following isomorphisms

$$
L P_{n}(\Psi)=(\mathbb{Z} / 2)^{2}, \mathbb{Z} / 2, \mathbb{Z} \oplus(\mathbb{Z} / 2)^{3},(\mathbb{Z} / 2)^{2}
$$

for $n=0,1,2,3 \bmod 4$, respectively. 
Theorem 6. For an inclusion $j: \mathbb{Z} / 4^{+} \rightarrow Q^{+,-}$, we have the following two-row diagram of L-groups

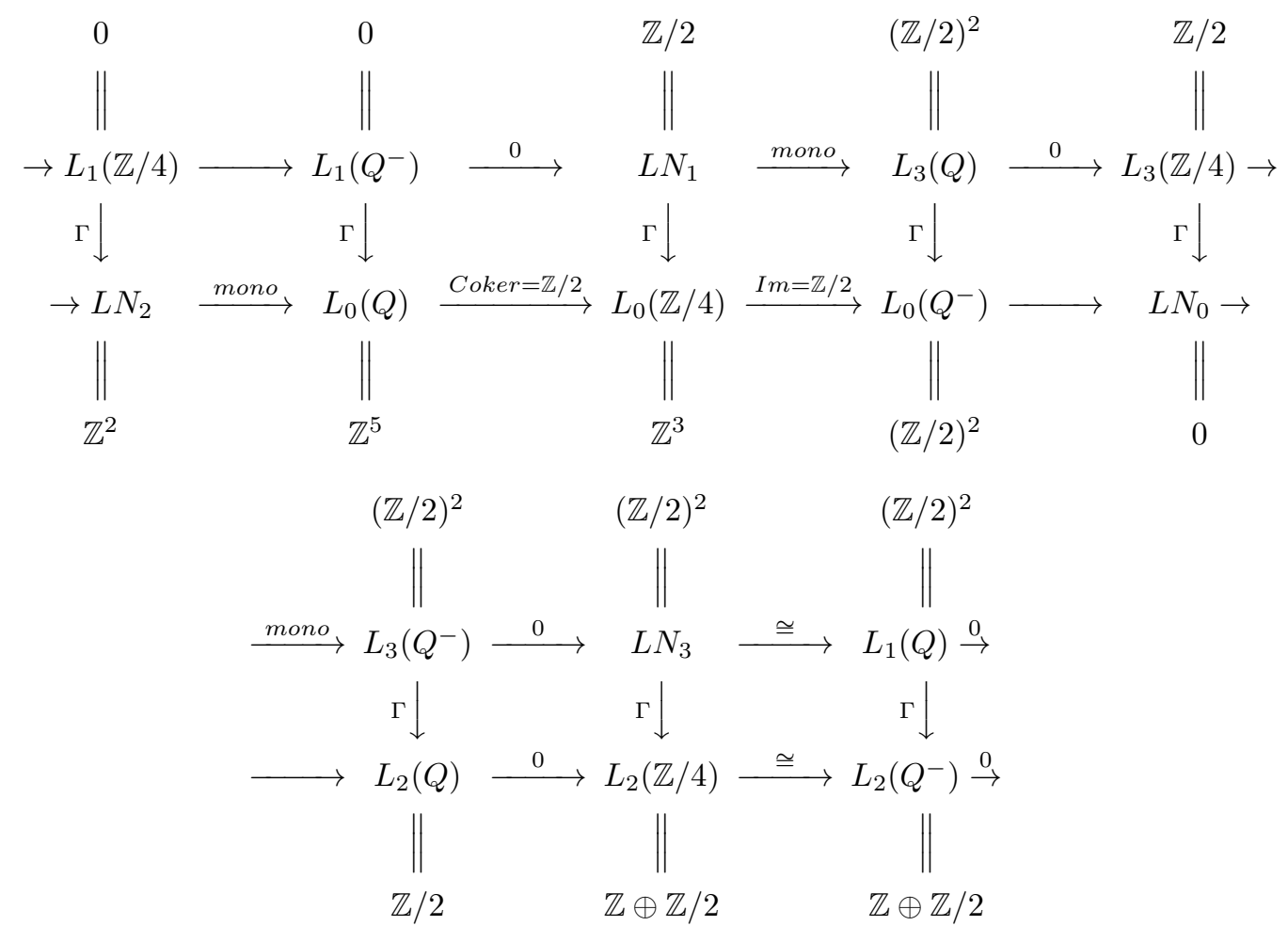

where $L N_{i}=L N_{i}\left(\mathbb{Z} / 4 \rightarrow Q^{+}\right) \cong L N_{i+2}\left(\mathbb{Z} / 4 \rightarrow Q^{-}\right)$.

Corollary 3. There exists an isomorphism

$$
L_{1}\left(Q^{+}\right) \cong \mathbb{Z} / 2 \oplus \mathbb{Z} / 2
$$

Corollary 4. Let $Y \subset X$ be be a Browder-Livesay pair of manifolds with a push-out square of fundamental groups

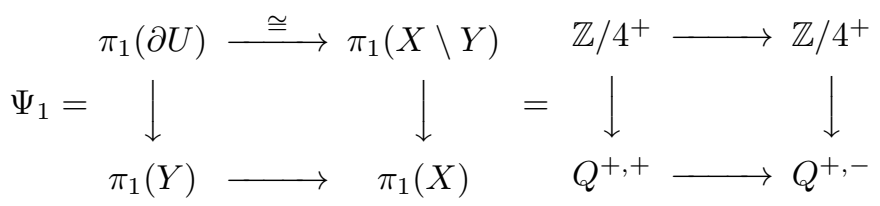

in the corresponding splitting problem. Then we have the following isomorphisms

$$
L P_{n}\left(\Psi_{1}\right)=\mathbb{Z}^{2}, \mathbb{Z} \oplus(\mathbb{Z} / 2)^{3},(\mathbb{Z} / 2)^{2},(\mathbb{Z} / 2)^{3}
$$

for $n=0,1,2,3 \bmod 4$, respectively. 
Theorem 7. For an inclusion $j: \mathbb{Z} / 4^{+} \rightarrow Q^{+}$, we have the following two-row diagram of L-groups

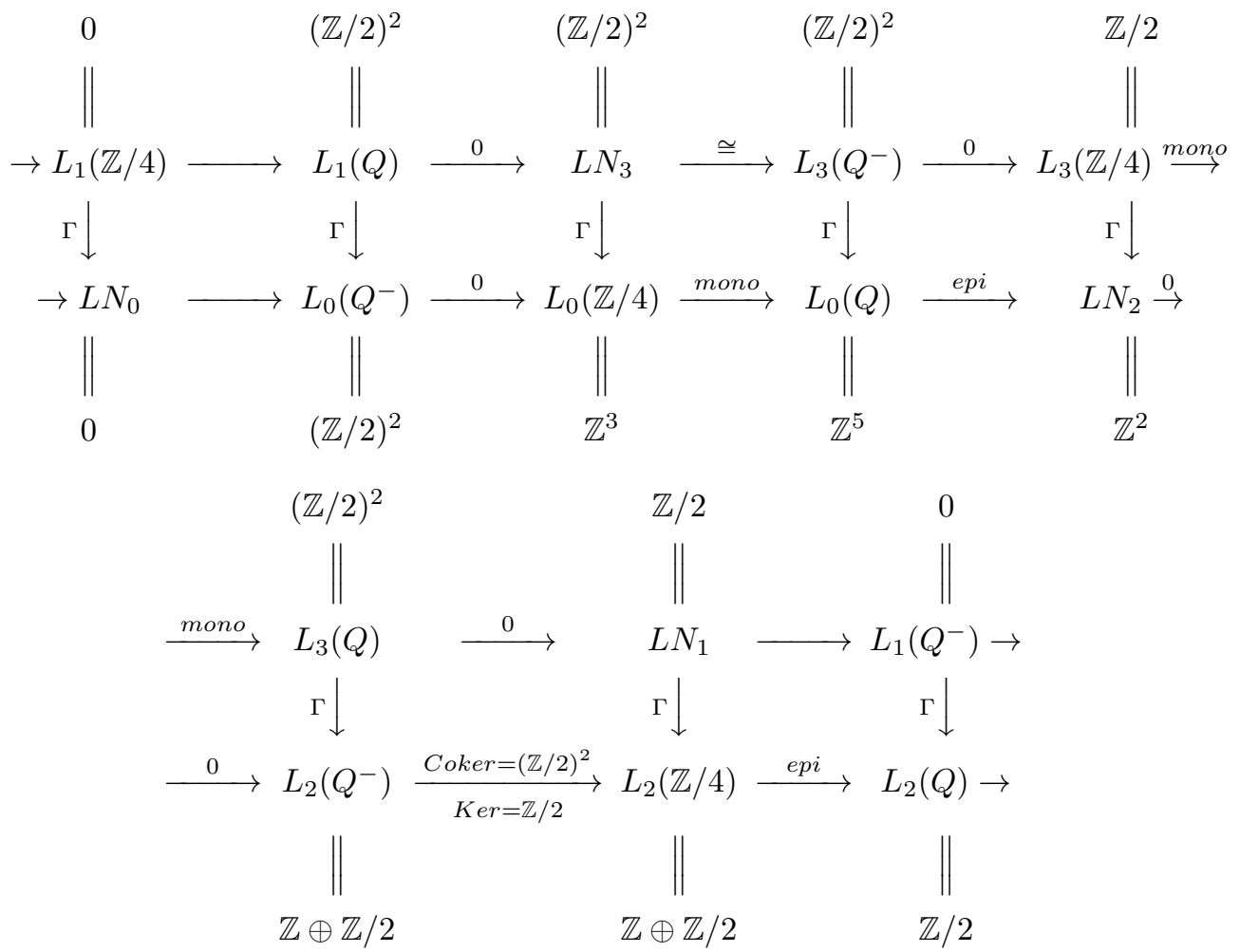

where $L N_{i}=L N_{i}\left(\mathbb{Z} / 4 \rightarrow Q^{+}\right) \cong L N_{i+2}\left(\mathbb{Z} / 4 \rightarrow Q^{-}\right)$.

Corollary 5. Let $Y \subset X$ be be a Browder-Livesay pair of manifolds with a push-out square of fundamental groups

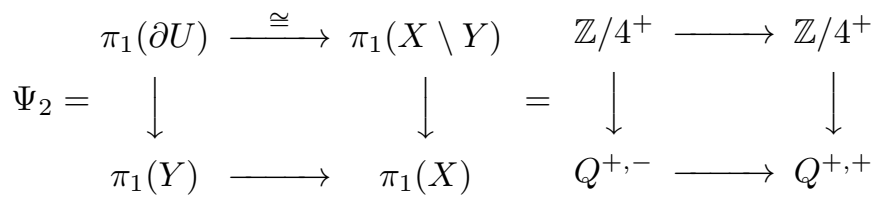

in the corresponding splitting problem. Then we have the following isomorphisms

$$
L P_{n}\left(\Psi_{2}\right)=(\mathbb{Z} / 2)^{2},(\mathbb{Z} / 2)^{2},(\mathbb{Z} / 2)^{2}, \mathbb{Z}^{3} \oplus(\mathbb{Z} / 2)^{2}
$$

for $n=0,1,2,3 \bmod 4$, respectively.

\section{REFERENCES}

1. S.E. Cappell, J.L. Shaneson, A counterexample on the oozing problem for closed manifolds, Lecture Notes in Math. 763 (1979), 627-634.

2. S.E. Cappell, J.L. Shaneson, Pseudo-free actions. I, Lecture Notes in Math. 763 (1979), 395-447.

3. I. Hambleton, Projective surgery obstructions on closed manifolds, Lecture Notes in Math. 967 (1982), 101-131.

4. I. Hambleton, A.F. Kharshiladze, A spectral sequence in surgery theory, Mat. Sbornik 183 (1992), 3-14; English transl. in Russian Acad. Sci. Sb. Math. 77 (1994), 1-9.

5. A.F. Kharshiladze, Iterated Browder-Livesay invariants and oozing problem, Mat. Zametki 41 (1987), 557-563; English transl. in Math. Notes 41 (1987), 312-315.

6. Yu.V. Muranov, Relative Wall groups and decorations, Mat. Sbornik 185 (1994), no. 12; English. transl. in Russian Acad. Sci. Sb. Math. 83 (1995), no. 2, 495-514. 
7. Yu.V. Muranov, D. Repovš, R. Jimenez, Surgery spectral sequence and manifolds with filtration, Trudy MMO 67 (2006), 294-325; English translation in Trans. Moscow Math. Soc. 67 (2006), 261-288.

8. A.A. Ranicki, The L-theory of twisted quadratic extensions, Can. J. Math. 39 (1987), 345-364.

9. C.T.C. Wall, Surgery on Compact Manifolds, Academic Press, London - New York, 1970; Second Edition (A. A. Ranicki, ed.), Amer. Math. Soc., Providence, R.I., 1999.

10. C.T.C. Wall, Classification of Hermitian forms. VI Group rings, Ann of Math. 103 (1976), 1-80.

Authors' addresses:

Friedrich Hegenbarth: Dipartimento di Matematica, Università di Milano, Via Saldini n. 50, 20133 Milano, Italy

E-mail: friedrich.hegenbarth@unimi.it

Yuri Muranov: Institute of Mathematics, Jagiellonian University, ul. prof. Stanislawa Lojasiewicza 6, 30-348 Krakow, Poland

E-mail: ymuranov@mail.ru

Dušan Repovš: Faculty of Mathematics ans Physics, and Faculty of Education, University of Ljubljana, Jadranska 19, 1000 Ljubljana, Slovenia;

E-mail: dusan.repovs@guest.arnes.si 